\title{
A NOTE ON PSEUDOCOMPACT GROUPS
}

\author{
ROBBERT FOKKINK
}

(Communicated by Jonathan M. Rosenberg)

\begin{abstract}
A question of W. W. Comfort and J. van Mill on pseudocompact groups is answered.
\end{abstract}

For each pair $X, Y$ of topological spaces let $C(X, Y)$ denote the set of all continuous maps from $X$ into $Y$. All topological spaces under consideration are assumed to be completely regular. Recall that a topological space is called pseudocompact if all continuous real-valued functions on $X$ are bounded.

Let $\Omega$ be a class of topological groups and $X$ a topological space. An element $G$ of $\Omega$ is called a free $\Omega$-group over $X$ if there exists a continuous map $l$ in $C(X, G)$ such that for every $H \in \Omega$ and $f \in C(X, H)$ there is a unique continuous homomorphism $F$ from $G$ into $H$ such that $F \circ l=f$. Note that a free $\Omega$-group is determined up to isomorphism. In the case that $\Omega$ is the class of all pseudocompact Abelian groups, $G$ is called the free pseudocompact group (over $X$ ). In their paper "On the existence of free topological groups", W. W. Comfort and J. van Mill showed that the free pseudocompact Abelian group over $X$ exists iff $X$ is empty.

Now W. W. Comfort and J. van Mill asked whether the weakly free pseudocompact Abelian group exists. This group is defined as the free pseudocompact Abelian group, but for the uniqueness condition on $F$, which is left out. It appears that this group also exists iff $X$ is empty.

Lemma 1. For every topological space $X$ the free compact Abelian group $F_{a} X$ exists.

Proof. This is an application of Freyd's lemma, see [3]. In fact one simply maps $X$ into the product of circle groups $T^{C(X, T)}$. Take $F_{a} X$ to be the closure of the group generated by the image of $X . F_{a} X$ has the desired properties since every compact Abelian group can be embedded as a subgroup in a product of circle groups.

Received by the editors October 21, 1988 and, in revised form, December 2, 1988.

1980 Mathematics Subject Classification (1985 Revision). Primary 22A05, 54B30, 54D30.

Key words and phrases. Free topological group, pseudocompact group. 
We have limited ourselves to completely regular topological spaces. This is not a heavy restriction. It has the advantage that $X$ can be identified with its image in $F_{a} X$.

Let $\langle X\rangle$ be the subgroup of $F_{a} X$ generated by $X$.

Lemma 2. $\langle X\rangle$ is not pseudocompact if $X$ is not empty.

Proof. Let $\zeta$ be an element of $T$ which is not a root of unity. Define $f: X \rightarrow T$ by $f(x)=\zeta$ for every $x \in X$. There exists a unique continuous homomorphism $F$ from $F_{a} X$ into $T$ which extends $f$. Thus $F$ maps $\langle X\rangle$ onto the subgroup $\langle\zeta\rangle$ generated by $\zeta$. Since $\langle\zeta\rangle$ is not pseudocompact, $\langle X\rangle$ cannot be pseudocompact.

Remember that a subset $Y$ of a topological space $X$ is called $G_{\delta}$-dense if every non-empty $G_{\delta}$ of $X$ intersects $Y$. A topological group $G$ is said to be totally bounded if, for each neighborhood $U$ of the identity, a finite number of translates of $U$ covers $G$. In [4] André Weil has shown that each totally bounded topological group $G$ can be embedded as a dense subgroup of a compact group. Furthermore this compact group is unique up to an isomorphism leaving $G$ pointwise fixed. As in [2] we call this compactification of $G$ the Weil-completion of $G$.

We will need the following characterization of pseudocompact groups, which can be found in [2].

Lemma 3. A totally bounded topological group is pseudocompact iff it is a $\boldsymbol{G}_{\delta^{-}}$ dense subgroup of its Weil-completion.

Now we can answer the question posed by W. W. Comfort and J. van Mill.

Theorem. The weakly free pseudocompact Abelian group over $X$ exists iff $X$ is empty.

Proof. Assume $X$ is topological space for which the weakly free pseudocompact Abelian group $P X$ exists.

Let $\Lambda$ be a set of cardinality $|\Lambda|>|P X| \cdot \omega$. Consider the totally bounded subgroup $A$ of $\left(F_{a} X\right)^{\Lambda}$ defined by $A=\left\{\left(x_{\lambda}\right)_{\lambda \in \Lambda} \mid x_{\lambda} \in F_{a} X, x_{\lambda}=0\right.$ for all but countably many $\lambda\}$. Note that every $G_{\delta}$ in $\left(F_{a} X\right)^{\Lambda}$ has a nonempty intersection with $A$. Since $\left(F_{a} X\right)^{\Lambda}$ is a compact group it must be the Weil-completion of $A$. Hence we conclude by Lemma 3 that $A$ is a pseudocompact group.

Let $\Delta$ be the diagonal of $\langle X\rangle$ in $\left(F_{a} X\right)^{\Lambda}, \Delta=\left\{\left(x_{\lambda}\right)_{\lambda \in \Lambda} \mid \exists x \in\langle X\rangle \forall \lambda \in \Lambda\right.$, $\left.x=x_{\lambda}\right\} . A+\Delta$ is pseudocompact since $A$ is pseudocompact.

Define $f: X \rightarrow\left(F_{a} X\right)^{\Lambda}$ by $f(x)=(x)_{\lambda \in \Lambda}$. Let $F$ be a continuous homomorphism from $P X$ into $A+\Delta$ which extends $f$. Consider the following subset of $\Lambda: K=\left\{\lambda \in \Lambda \mid \exists p \in P X,\left(\pi_{\lambda} \circ F\right)(p) \notin\langle X\rangle\right\} . \quad \pi_{\lambda}$ denotes the projection on the coordinate $\lambda$. By the definition of $A$ we have that for every $p \in P X,\left(\pi_{\lambda} \circ F\right)(p)$ is an element of $\langle X\rangle$ for all but countably many $\lambda$. Hence $|K| \leq|P X| \cdot \omega<|\Lambda|$. So there has to be a $\kappa$ in $\Lambda$ which is not an element of $K$. 
Now $\pi_{\kappa} \circ F(P X)$ is contained in $\langle X\rangle$. On the other hand since $\pi_{\kappa} \circ f(X)=X$ the two groups have to be equal. $\pi_{\kappa} \circ F(P X)=\langle X\rangle$. We conclude $\langle X\rangle$ is pseudocompact. Then $X$ has to be empty as follows from Lemma 2.

\section{REFERENCES}

1. W. W. Comfort and Jan van Mill, On the existence of free topological groups, Top. and its Appl. 29 (1988), 245-265.

2. W. W. Comfort and Kenneth A. Ross, Pseudocompactness and uniform continuity in topological groups, Pacific J. Math. 16 (1966), 483-496.

3. S. MacLane, Categories for the working mathematician, New York, 1971.

4. André Weil, Sur les espaces à structure uniforme et sur la topologie générale, Publ. Math. Univ. Strasbourg, Hermann, Paris, 1937.

Technical University Delft, Postbus 356, 2600 AJ Delft, Netherlands 